\title{
Prognostic four-gene signature for overall survival in patients with hepatocellular carcinoma
}

\author{
Tatsuo Kanda $^{1}$ (i) $\cdot$ Osamu Yokosuka ${ }^{2} \cdot$ Mitsuhiko Moriyama $^{1}$
}

Received: 10 July 2019 / Accepted: 30 July 2019 / Published online: 16 August 2019

(c) Asian Pacific Association for the Study of the Liver 2019

Hepatocellular carcinoma (HCC) is one of the human cancers with a poor long-term prognosis worldwide. Exosomes could mediate communication between both cancer and non-cancer cells [1]. Recent research demonstrated that proteins, DNAs, and various forms of RNA such as microRNA (miRNA), long noncoding RNA (lncRNA), and messenger RNA (mRNA) transferred by exosomes, all contribute to the development of HCC [1].

In this issue, Yan et al. performed integrated competing endogenous RNA (ceRNA) network analysis and established the prognostic signature with the least absolute shrinkage and selection operator algorithm [2]. They used high-throughput sequencing data of mRNAs, lncRNAs, miRNAs of $374 \mathrm{HCC}$ samples, and 50 normal samples from the Cancer Genome Atlas (TCGA, https://portal.gdc.cancer.gov/) and performed multivariate Cox regression analysis to identify independent factors for the prediction of overall survival (OS) of patients with HCC [2]. They confirmed their results by external validation cohorts from two independent centers [2].

Among the TCGA set, they identified a total of 1993 differentially expressed mRNAs, of which 1788 (89.71\%) were upregulated and 205 (10.29\%) were downregulated mRNAs [2]. Although they found 1071 differentially expressed lncRNAs, including 1014 (94.67\%) upregulated and 57 (5.32\%) downregulated lncRNAs, only 162 (95.29\%) upregulated and $8(4.71 \%)$ downregulated differentially expressed miRNAs were observed [2]. Next, by performing functional enrichment and survival analyses of key ceRNAs, they identified 20 mRNAs, 1 miRNA (miR-137) and 14 lncRNAs were significantly associated with the OS of patients with HCC [2].

Tatsuo Kanda

kanda.tatsuo@nihon-u.ac.jp; kanda2t@yahoo.co.jp

1 Division of Gastroenterology and Hepatology, Department of Medicine, Nihon University School of Medicine, 30-1 Oyaguchi-kamicho, Itabashi-ku, Tokyo 173-8610, Japan

2 Department of Gastroenterology and Nephrology, Chiba University Graduate School of Medicine, Chiba, Japan
A LASSO regression model [3] was used to identify a predictive signature from a TCGA training set and four genes [PDZ-binding kinase (PBK), Chromobox 2 (CBX2), CLSPN, and cytoplasmic polyadenylation element-binding protein 3 (CPEB3)], associated with the OS of patients with HCC, were identified [2]. They also established a prognostic risk score and validated the risk score in their HCC patients [2]. The signature of these four genes was an independent prognostic factor particularly for Asian patients with serum $\alpha$-fetoprotein (AFP) $\geq 20 \mathrm{ng} / \mathrm{ml}$ [2].

PBK has been implicated in the malignancy formation in cancers, including hepatocellular carcinoma $[4,5]$. PBK is significantly upregulated in HCC tissue and is associated with increased vascular invasion and shorter survival [5]. PBK enhances the promotion of metastasis of HCC by activating the ETS variant 4 (ETV4)-urokinase plasminogen activator receptor (UPAR) signaling pathway and could be a promising diagnostic biomarker and a potential therapeutic target for HCC metastasis [5].

CBX2 is highly expressed in human HCC tissues and is associated with poor prognosis in patients with $\mathrm{HCC}$ [6]. CBX2 is a crucial component of the polycomb group complex, polycomb repressive complex 1 (PRC1), which is involved in human carcinogenesis [6].

Claspin, which is encoded by CLSPN, is a checkpoint adapter and DNA replication factor. Claspin degradation is tightly regulated via ubiquitination with multiple ubiquitin ligases and de-ubiquitinases at the proteasome [7]. Claspin contributes to the regulation of checkpoint proficiency and plays a role in the termination of checkpoint-mediated cell cycle arrest. Claspin expression abbreviations and its mutations are clearly associated with both familial and sporadic cancers [7]. Claspin overexpression is one of the markers of radio-resistance in metastasis lung cancer [8].

Zhou et al. demonstrated that miR-107 may facilitate HCC pathogenesis through the CPEB3/epidermal growth factor receptor (EGFR) axis [9]. Downregulation of CPEB3 promotes human HCC cell proliferation and 


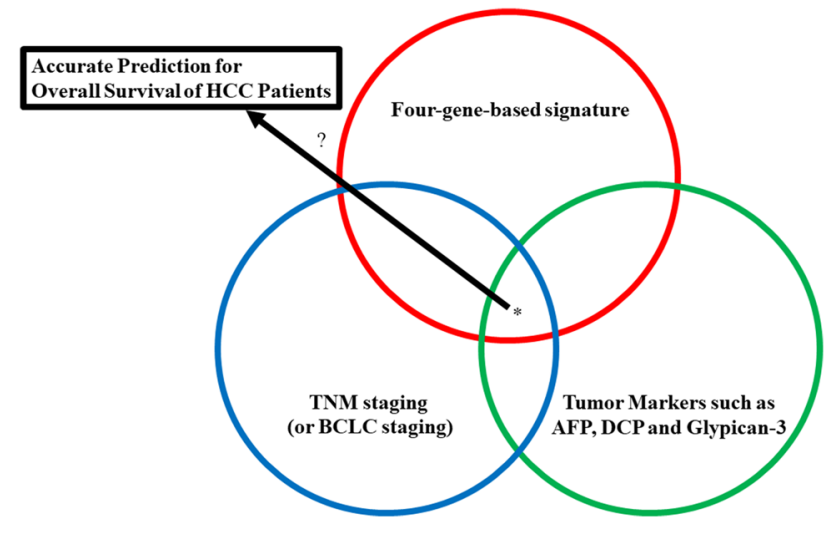

Fig. 1 Future perspective of accurate prediction for overall survival (OS) of patients with hepatocellular carcinoma (HCC) in AsiaPacific region. Four-gene-based-signature [PDZ-binding kinase (PBK), Chromobox 2 (CBX2), CLSPN and cytoplasmic polyadenylation element binding protein 3 (CPEB3)], TNM TNM classification of malignant tumors, $B C L C$ barcelona clinic liver cancer, $A F P$ $\alpha$-fetoprotein, $D C P$ des- $\gamma$-carboxy prothrombin

metastasis [9]. miR-107 was involved in various pathological processes including carcinogenesis, and high expression of miR-107 was observed in HCC tissues compared with non-HCC tissues [9]. Although PBK, CBX2, and CLSPN are located in nucleus, CPEB3 is located in plasma membrane and cytoplasm as well as nucleus. Unfortunately, the study conducted by Yan et al. did not include an analysis of protein expression [2].

Nonetheless, a novel lncRNAs-miRNAs-mRNAs ceRNA network in HCC based on genome-wide analysis may be useful for finding a new candidate therapeutic decision marker based on the ceRNA network in the prediction of OS of patients with HCC [2]. Further studies will be needed to identify the underlying mechanisms involving these prognostic four genes, miRNAs and lncRNAs. Integrated bioinformatic analysis of mRNA, miRNA, and lncNA expressions, genetic mutations, and epigenetic modifications associated with HCC development will provide important new information in this area. In Asia-Pacific region, more translational research should be performed to reveal which is more accurate way to predict the OS by the four-gene-based signature with or without the biological markers such as AFP, des- $\gamma$-carboxy prothrombin (DCP), and glypican-3 (Fig. 1) [2, 10, 11].

Funding None.

\section{Compliance with ethical standards}

Conflict of interest All authors have declared that they have no conflicts of interest.
Ethical approval This article does not contain any studies with human participants or animals performed by any of the authors.

Informed consent Not necessary, see above.

\section{References}

1. Sasaki R, Kanda T, Yokosuka O, Kato N, Matsuoka S, Moriyama M. Exosomes and hepatocellular carcinoma: from bench to bedside. Int J Mol Sci 2019;20:E1406. https://doi.org/10.3390/ijms2 0061406 (PMID: 30897788)

2. Yan Y, Lu Y, Mao K, Zhang M, Liu H, Zhou Q, et al. Identification and validation of prognostic 4 genes signature for hepatocellular carcinoma: integrated ceRNA network analysis. Hepatol Int 2019. https://doi.org/10.1007/s12072-019-09962-3 (Epub ahead of print, PMID: 31321712)

3. Shiino S, Matsuzaki J, Shimomura A, Kawauchi J, Takizawa S, Sakamoto H, et al. Serum miRNA-based prediction of axillary lymph node metastasis in breast cancer. Clin Cancer Res 2019;25:1817-1827. https://doi.org/10.1158/1078-0432.ccr-181414 (PMID: 30482779)

4. Yang YF, Pan YH, Cao Y, Fu J, Yang X, Zhang MF, et al. PDZ binding kinase, regulated by FoxM1, enhances malignant phenotype via activation of $\beta$-catenin signaling in hepatocellular carcinoma. Oncotarget 2017;8:47195-47205. https://doi.org/10.18632 /oncotarget.17587 (PMID: 28525379)

5. Yang QX, Zhong S, He L, Jia XJ, Tang H, Cheng ST, et al. PBK overexpression promotes metastasis of hepatocellular carcinoma via activating ETV4-uPAR signaling pathway. Cancer Lett 2019;452:90-102. https://doi.org/10.1016/j.canlet.2019.03.028 (PMID: 30914208)

6. Mao J, Tian Y, Wang C, Jiang K, Li R, Yao Y, et al. CBX2 Regulates proliferation and apoptosis via the phosphorylation of YAP in hepatocellular carcinoma. J Cancer 2019;10:2706-2719. https ://doi.org/10.7150/jca.31845 (PMID: 31258779)

7. Smits VAJ, Cabrera E, Freire R, Gillespie DA. Claspin-checkpoint adaptor and DNA replication factor. FEBS J 2019;286:441455. https://doi.org/10.1111/febs.14594 (PMID: 29931808)

8. Choi SH, Yang H, Lee SH, Ki JH, Nam DH, Yoo HY. TopBP1 and claspin contribute to the radioresistance of lung cancer brain metastases. Mol Cancer 2014;13:211. https://doi. org/10.1186/1476-4598-13-211 (PMID: 25216549)

9. Zou CD, Zhao WM, Wang XN, Li Q, Huang H, Cheng WP, et al. MicroRNA-107: a novel promoter of tumor progression that targets the CPEB3/EGFR axis in human hepatocellular carcinoma. Oncotarget 2016;7:266-278. https://doi.org/10.18632/oncotarget .5689 (PMID: 26497556)

10. Murata K, Saito A, Katagiri S, Ariizumi S, Nakano M, Yamamoto M. Association of des- $\gamma$-carboxy prothrombin production and sonazoid-enhanced ultrasound findings in hepatocellular carcinomas of different histologic grades. J Med Ultrason (2001) 2018;45:223-229. https://doi.org/10.1007/s10396-017-0816-3 (PMID: 28828695)

11. Hamaoka M, Kobayashi T, Tanaka Y, Mashima H, Ohdan H. Clinical significance of glypican-3-positive circulating tumor cells of hepatocellular carcinoma patients: a prospective study. PLoS One 2019;14:e0217586. https://doi.org/10.1371/journal.pone.0217586 (PMID: 31141571)

Publisher's Note Springer Nature remains neutral with regard to jurisdictional claims in published maps and institutional affiliations. 